\title{
EXPLICIT DETERMINATION OF PINNED-PINNED BEAMS WITH A FINITE NUMBER OF GIVEN BUCKLING LOADS
}

\author{
ANTONINO MORASSI \\ Polytechnic Department of Engineering and Architecture, University of Udine, Via Cotonificio \\ 114 \\ Udine, 33100, Italy* \\ antonino.morassi@uniud.it \\ MARTINA PRESSACCO \\ Polytechnic Department of Engineering and Architecture, University of Udine, Via Cotonificio \\ 114 \\ Udine, 33100, Italy \\ martina.pressacco@libero.it
}

ANASTASIA VRECH

Polytechnic Department of Engineering and Architecture, University of Udine, Via Cotonificio 114

Udine, 33100, Italy

vrech.anastasia@spes.uniud.it

Received (Day Month Year)

Accepted (Day Month Year)

\begin{abstract}
We present an analytical procedure for the exact, explicit construction of Euler-Bernoulli beams with given values of the first $N$ buckling loads. The result is valid for pinnedpinned end conditions and for beams with regular bending stiffness. The analysis is based on a reduction of the buckling problem to an eigenvalue problem for a vibrating string, and uses recent results on the exact construction of Sturm-Liouville operators with prescribed natural frequencies.
\end{abstract}

Keywords: Buckling loads; beams; inverse problems.

\section{Introduction}

The problem of determining the buckling load of a compressed beam is of importance in several fields of Structural Mechanics. When formulated within the linearized equilibrium theory for Euler-Bernoulli-Kirchhoff bending beams, the buckling problem is equivalent to determining the eigenvalues $P_{m}, m \geq 1$, and, in particular, the smallest eigenvalue $P_{1}$, and the buckling modes $v_{m}=v_{m}(x)$ of the

* Corresponding author: Polytechnic Department of Engineering and Architecture, University of Udine, Via Cotonificio 114, 33100 Udine, Italy. Tel. +39 0432558739; fax +390432558700. 
fourth order differential operator

$$
\frac{d^{2}}{d x^{2}}\left(E I(x) \frac{d^{2} v_{m}(x)}{d x^{2}}\right)+P_{m} \frac{d^{2} v_{m}(x)}{d x^{2}}=0, \quad x \in(0, L),
$$

where $E I(x)$ is the bending stiffness of beam's cross-section. Within the forward approach to the buckling problem, the coefficient $E I(x)$ is given and standard analytical/numerical methods can be used to find the eigenpairs of (1.1), under a specified set of end conditions.

In addition to the direct approach, there are situations important in real-world applications in which an inverse approach to the buckling problem is adopted. One class of problems is concerned with optimal buckling design, namely the determination of the maximum values of the smallest buckling load $P_{1}$ for a given structural weight, or alternatively, the minimization of the structural weight that satisfies a prescribed smallest buckling load. The problem was originally posed by Lagrange and a large number of publications have appeared on this topic, see, among others, the classical Refs. 1, 2, 3, 4, 5, 6 .

A second class of inverse-like applications is concerned with the construction of beams having specified buckling properties. This paper belongs to this second class of applications. Specifically, here we show how to explicitly construct families of bending coefficients $E I(x)$ such that the eigenvalue problem (1.1) has exactly given values of the first $N$ buckling loads $\left\{P_{m}\right\}_{m=1}^{N}, 1 \leq N<\infty$, under pinned-pinned end conditions, e.g., $v_{m}=\frac{d^{2} v_{m}}{d x^{2}}=0$ at $x=0$ and $x=L$.

From the point of view of Structural Mechanics, the construction of beams with given first smallest buckling load (e.g., $N=1$ ) is obviously the case of main practical interest. However, some authors have demonstrated that optimal buckling design could be multi-modal, namely, the optimal solutions can have a double eigenvalue, corresponding to two distinct buckling modes ${ }^{4}$. Therefore, the multiplicity of the eigenvalue may result in a complicated buckling mode shape. This undesirable behavior can be avoided by imposing sufficiently separated buckling loads and, with this aim, the specification of higher buckling loads (such as the second buckling load, for example) can be of interest in practical applications.

It should be also noticed the analytical approach we adopted provides exact closed form solution to the inverse problem, that is, the bending stiffness coefficient of the beams having given values of the first $N$ buckling loads is reconstructed by means of closed form expressions. As by-product of the analysis, we have obtained exact closed form expressions of the buckling modes for pinned-pinned beams having variable bending stiffness. The determination of closed-form buckling eigensolutions is an important topic in Structural Mechanics; see, for example, the research developed in Refs. 7, 8 (columns and beams on elastic foundation with trigonometric variability both of axial loads and bending stiffness) and Ref. 9 (buckling analysis of columns with variable cross-section via the Effective Stiffness Method). The new exact buckling solutions found in this paper can be useful both for testing the accuracy of numerical discrete models and for the formulation of structural opti- 
mization problems (particularly, for $N=1$ ), such as the determination of beams having maximum global stiffness or satisfying certain strength/strain requirements.

From the analytical point of view, our method is based on a reduction of the buckling problem to an equivalent eigenvalue problem for a class of taut strings with fixed ends, and adapts recent results on the exact construction of secondorder Sturm-Liouville operators in canonical form with prescribed natural frequencies (see Ref. 10). In particular, the key mathematical tool of our analysis relies on a classical lemma by Darboux ${ }^{11}$. This lemma allows to explicitly construct families of Sturm-Liouville operators that share all the eigenvalues of a given operator, with the exception of a single eigenvalue which is free to move in a prescribed interval. These operators are called quasi-isospectral operators. Moreover, the Darboux lemma is applied once again in iterated form to determine strings corresponding to the quasi-isospectral Sturm-Liouville operators and, ultimately, to find beams quasi-isobuckling to a given beam, under pinned-pinned end conditions.

Finally, it is appropriate to compare the results of this paper and those obtained in Ref. 12. In Ref. 12, the authors showed how to construct families of EulerBernoulli beams which have exactly the same infinite sequence of buckling loads of a given beam under a specified set of end conditions. These beams were called isobuckling beams. Here, instead, we show how to construct a beam with given values of (a finite number of) buckling loads, and the crucial point is the ability to construct quasi-isobuckling beams, that is beams having all the buckling loads in common, with the exception of a single one. Although the spectral equivalence with a class of strings and the Darboux lemma are important points of both the present analysis and of that developed in Ref. 12, the results found here are clearly different and, in some respects, are more general than those obtained in the mentioned paper. In fact, the procedure for the construction of isobuckling beams proposed in Ref. 12 cannot be used to construct beams having prescribed values of the first $N$ buckling loads.

The plan of the paper is as follows. In Section 2 we recall the equivalence between the buckling problem and the eigenvalue problem for a vibrating string. The main steps of the construction procedure are presented in Section 3. Examples of quasiisobuckling beams are illustrated in Section 4. The Darboux Lemma is recalled in the Appendix.

\section{Elastic buckling of a beam and an equivalent string problem}

Consider a thin straight elastic beam under constant compressive axial load $P$, $P>0$. The buckling problem is governed by the Euler-Bernoulli-Kirchhoff equation (see Ref. 13)

$$
\frac{d^{2}}{d x^{2}}\left(E I(x) \frac{d^{2} v(x)}{d x^{2}}\right)+P \frac{d^{2} v(x)}{d x^{2}}=0, \quad x \in(0, L)
$$


where $v=v(x)$ is the transverse displacement of the beam axis at the cross-section of abscissa $x$ evaluated with the principal plane of bending. In equation (2.1), $E$ is the Young's modulus of the material, $E=$ const. $>0$, and $I=I(x)$ is the second moment of the cross-sectional area about a principal axis through the centroid of the cross-section. We shall be concerned with beams for which $I(x)$ is a strictly positive, twice continuously differentiable function of $x$ in $[0, L]$, e.g.

$$
I(x) \geq I_{0}>0, \quad x \in[0, L], \quad I \in C^{2}([0, L]) .
$$

Let us assume that the beam has Pinned-Pinned (P-P) ends. The buckling problem consists in solving the eigenvalue problem

where

$$
\left\{\begin{array}{l}
\frac{d^{2}}{d x^{2}}\left(I(x) \frac{d^{2} v(x)}{d x^{2}}\right)+\lambda^{2} \frac{d^{2} v(x)}{d x^{2}}=0, \quad x \in(0, L), \\
v(0)=\frac{d^{2} v(0)}{d x^{2}}=0, \\
v(L)=\frac{d^{2} v(L)}{d x^{2}}=0,
\end{array}\right.
$$

$$
\lambda^{2}=\frac{P}{E} .
$$

Under the above assumptions, there exists an infinite sequence of buckling loads $\left\{P_{m}=\lambda_{m}^{2} E\right\}_{m=1}^{\infty}$, with

$$
0<P_{1}<P_{2}<\ldots, \quad \lim _{m \rightarrow \infty} P_{m}=\infty,
$$

such that (2.3)-(2.5) have a non-trivial solution $v_{m}=v_{m}(x), m \geq 1$. This sequence is the buckling spectrum of the Pinned-Pinned beam and we write

$$
\left\{\lambda_{m}^{2}\right\}_{m=1}^{\infty}=\operatorname{BSp}(I(x) ; P-P) .
$$

The following proposition states the equivalence between the eigenvalue problem (2.3)-(2.5) and the free vibration problem for a family of taut strings.

Proposition 2.1 If $\left\{\lambda^{2}, v(x)\right\}$ is an eigenpair of (2.3)-(2.5) with $I=I(x)$ satisfying (2.2), then $\left\{\lambda^{2}, v(x)\right\}$ is an eigenpair of

with

$$
\left\{\begin{array}{l}
\frac{d^{2} v(x)}{d x^{2}}+\lambda^{2} \rho(x) v(x)=0, \quad x \in(0, L), \\
v(0)=0=v(L),
\end{array}\right.
$$

$$
\rho(x)=\frac{1}{I(x)}, \quad x \in[0, L] .
$$

Viceversa, if $\left\{\lambda^{2}, v(x)\right\}$ is an eigenpair of (2.9)-(2.10), then $\left\{\lambda^{2}, v(x)\right\}$ is an eigenpair of (2.3)-(2.5).

The eigenvalue problem (2.9)-(2.10) describes the free, infinitesimal, transverse vibration of amplitude $v=v(x)$ of a string with frequency $\lambda$ and mass density $\rho=\rho(x), \rho \in C^{2}([0, L])$ and $\rho(x) \geq \rho_{0}>0$ in $[0, L]$. The string is pulled with unit tension, has length $L$ and is fixed at both ends. A proof of Proposition 2.1 is presented in Ref. 12 (Proposition 1). 


\section{Construction of beams with given buckling loads}

Let $n, n \geq 1$, be given. The key step of our method is based on the explicit construction of a new P-P beam quasi-isobuckling to the given beam, that is a beam $I=I(x)$ having the same buckling loads as the given beam $\widehat{I}=\widehat{I}(x)$, with the exception of the $n$th buckling load. In fact, by keeping fixed all the eigenvalues $\lambda_{m}^{2}$ with $m \neq n$ and moving the $n$th eigenvalue $\lambda_{n}^{2}$ to the desired value, say $\widetilde{\lambda}_{n}^{2}$, and using repeatedly the procedure, after $N$ steps we will construct a beam with the first $N$ given eigenvalues $\left\{\widetilde{\lambda}_{m}^{2}\right\}_{m=1}^{N}$, and the construction is completed.

The main steps of the construction of P-P beams $I=I(x)$ quasi-isobuckling to a given $\mathrm{P}-\mathrm{P}$ beam $\widehat{I}=\widehat{I}(x)$ are the following.

STEP 1. The string eigenvalue problem (2.9)-(2.10) is reduced to SturmLiouville canonical form with Schrödinger potential $\widehat{q}$.

Step 2. The Darboux Lemma (see Appendix) is used to construct explicit families of Schrödinger potentials $q$ quasi-isospectral to the initial potential $\widehat{q}$.

STEP 3. The Darboux Lemma is applied once more in iterate form to determine string mass densities corresponding to the quasi-isospectral potentials $q$.

SteP 4. Finally, the equivalence stated in Proposition 2.1 is used to find P-P beams $I=I(x)$ quasi-isobuckling to the initial P-P beam $\widehat{I}=\widehat{I}(x)$.

We shall analyze Steps 1-4 in the following subsections.

\subsection{Reduction to canonical form.}

Suppose that a P-P beam $\widehat{I}=\widehat{I}(x)$, satisfying conditions $(2.2)$, is given. The buckling spectrum of this beam is $\left\{\widehat{\lambda}_{m}^{2}\right\}_{m=1}^{\infty}=\operatorname{BSp}(\widehat{I}(x) ; P-P)$. Denote by $\{\widehat{\rho}(x)\}$ the corresponding Fixed-Fixed (F-F) string as defined in Proposition 2.1, with spectrum $\left\{\widehat{\lambda}_{m}^{2}\right\}_{m=1}^{\infty}=\operatorname{Sp}(\widehat{\rho}(x) ; F-F)$. The Liouville transformation

$$
\begin{gathered}
\xi(x)=\frac{1}{\widehat{p}} \int_{0}^{x}(\widehat{\rho}(s))^{1 / 2} d s, \quad \widehat{p}=\int_{0}^{L}(\widehat{\rho}(s))^{1 / 2} d s, \\
y(\xi)=\widehat{a}(\xi) v(x), \quad \widehat{a}^{4}(\xi)=\frac{L^{2}}{\widehat{p}^{2}} \widehat{\rho}(x),
\end{gathered}
$$

reduces the eigenvalue problem (2.9)-(2.10) (with $\rho$ replaced by $\widehat{\rho}$ ) for $\left\{\widehat{\lambda}^{2}, v(x)\right\}$ to the Sturm-Liouville canonical form

$$
\left\{\begin{array}{l}
\frac{d^{2} y(\xi)}{d \xi^{2}}+\widehat{\mu} y(\xi)=\widehat{q}(\xi) y(\xi), \quad \xi \in(0,1), \\
y(0)=0=y(1)
\end{array}\right.
$$

where the eigenvalue $\widehat{\mu}$ and the potential $\widehat{q}(\xi), \widehat{q} \in C^{0}([0,1])$, are defined as

$$
\widehat{\mu}=\widehat{p}^{2} \widehat{\lambda}^{2}, \quad \widehat{q}(\xi)=\frac{1}{\widehat{a}(\xi)} \frac{d^{2} \widehat{a}(\xi)}{d \xi^{2}}, \quad \xi \in(0,1) .
$$


6 Morassi, Pressacco and Vrech

\subsection{Quasi-isospectral potentials.}

Following the analysis developed in Ref. 14, it is possible to explicitly construct families of Sturm-Liouville operators $L=-\frac{d^{2}}{d \xi^{2}}+q(\xi)$, with potential $q(\xi)$ quasiisospectral to the potential $\widehat{q}(\xi)$ under Dirichlet end conditions. The analysis is based on the Darboux Lemma described in the Appendix. Here, we simply recall the main result. Let us introduce some notation. Let $n, n \geq 1$, be a given number and let $t \in \mathbb{R}$ be such that

$$
\mu_{n-1}(\widehat{q})<\mu_{n}(\widehat{q})+t<\mu_{n+1}(\widehat{q}),
$$

with $\mu_{0}(\widehat{q})=0$. Denote by $\delta_{i j}$ the Kronecker symbol. For $\mu \in \mathbb{C}$, let $y_{i}=y_{i}(\xi, \widehat{q}, \mu)$, $i=1,2$, be the solution to the initial value problem

$$
\left\{\begin{array}{l}
y_{i}^{\prime \prime}+\mu y_{i}=\widehat{q} y_{i}, \quad \xi \in(0,1), \\
y_{i}(0)=\delta_{i 1}, \\
y_{i}^{\prime}(0)=\delta_{i 2},
\end{array}\right.
$$

and denote by $w_{n}=w_{n}(\xi, \widehat{q}, \mu)$ the solution to

$$
\left\{\begin{array}{l}
w_{n}^{\prime \prime}+\mu w_{n}=\widehat{q} w_{n}, \quad \xi \in(0,1), \\
w_{n}(0)=1 \\
w_{n}(1)=y_{1}\left(1, \mu_{n}, \widehat{q}\right),
\end{array}\right.
$$

for $\mu \neq \mu_{n}$ (note that the function $w_{n}$ has a removable singularity at $\mu=\mu_{n}$ ). Let

$$
\omega_{n}(\xi, \widehat{q}, \mu)=w_{n}(\xi, \widehat{q}, \mu) \frac{d z_{n}(\xi, \widehat{q})}{d \xi}-\frac{d w_{n}(\xi, \widehat{q}, \mu)}{d \xi} z_{n}(\xi, \widehat{q}) \equiv\left[w_{n}, z_{n}\right](\xi),
$$

where $z_{n}$ is the $n$th eigenfunction of $(3.3)-(3.4)$. For every $\widehat{q} \in C^{0}([0,1])$, the function $\omega_{n}=\omega_{n}(\xi, \widehat{q}, \mu), n \geq 1$, is a continuous and strictly positive function on $[0,1] \times\left(\mu_{n-1}(\widehat{q}), \mu_{n+1}(\widehat{q})\right)$. Moreover, $\omega_{n}$ is a $C^{2}$-function of the variable $\xi$ in $[0,1]$ (see Ref. 14). We define $w_{n, t}=w\left(\xi, \widehat{q}, \mu_{n}+t\right)$ and $\omega_{n, t}=\omega\left(\xi, \widehat{q}, \mu_{n}+t\right)$.

Under the above notation, for every given $n, n \geq 1$, and $t$ satisfying (3.6), it is possible to prove that the potential

$$
q(\xi)=\widehat{q}(\xi)-2 \frac{d^{2}}{d \xi^{2}}\left(\ln \omega_{n, t}(\xi)\right)
$$

has all the same eigenvalues of the potential $\widehat{q}(\xi)$, with the exception of the $n$th eigenvalue, which takes the value $\mu_{n}(q)=\mu_{n}(\widehat{q})+t$. Moreover, the eigenfunctions $\left\{k_{m, t}\right\}_{m=1}^{\infty}$ associated to $q(\xi)$ have the following explicit expressions

$$
\begin{gathered}
k_{m, t}=z_{m}-t \frac{w_{n, t}}{\omega_{n, t}} \int_{0}^{\xi} z_{m}(s) z_{n}(s) d s, \quad \text { for } m \geq 1, m \neq n \\
k_{n, t}=\frac{z_{n}}{\omega_{n, t}} .
\end{gathered}
$$




\subsection{Quasi-isospectral strings.}

The eigenvalues $\left\{\widehat{\mu}_{m}\right\}$ of $(3.3)-(3.4)$ have the asymptotic form

$$
\widehat{\mu}_{m}=(m \pi)^{2}+\widehat{O}(1), \quad \text { as } m \rightarrow \infty,
$$

with $\widehat{O}(1)$ bounded quantity as $m \rightarrow \infty$. Therefore, if the two strings $\{\widehat{\rho}(x)\}$ and $\{\rho(x)\}$ are quasi-isospectral, i.e. $\widehat{\lambda}_{m}^{2}=\lambda_{m}^{2}$ for every $m \neq n$, where $n \geq 1$ is a given number, then, for $m$ large,

$$
\widehat{p}^{2} \widehat{\lambda}_{m}^{2}=(m \pi)^{2}+\widehat{O}(1), \quad p^{2} \lambda_{m}^{2}=(m \pi)^{2}+O(1),
$$

so that

$$
\widehat{p}^{2}=p^{2}
$$

Now, to find a supported string $\{\rho(x)\}$ quasi-isospectral to a given supported string $\{\widehat{\rho}(x)\}$, we must preliminarily find a function $a=a(\xi)$ corresponding to the new quasi-isospectral potential $q=q(\xi)$ given by (3.14), that is

$$
\frac{d^{2} a(\xi)}{d \xi^{2}}=q(\xi) a(\xi)
$$

with $a=a(\xi)$ of one-sign in [0,1]. A double application of the Darboux Lemma yields the following explicit expression for $a$ :

$$
a(\xi)=\widehat{a}(\xi)-t \frac{w_{n, t}(\xi)}{\mu_{n} \omega_{n, t}(\xi)}\left[z_{n}, \widehat{a}\right](\xi), \quad n \geq 1,
$$

see Ref. 10 for details. In particular, it is possible to prove that $a=a(\xi)$ given by (3.21) is a $C^{2}$-function of one sign in $[0,1]$ for every $t$ satisfying (3.6).

To complete the construction of quasi-isospectral strings, we reverse the Liouville transformation (3.1)-(3.2), namely

$$
\begin{gathered}
x(\xi)=\frac{L}{K} \int_{0}^{\xi} \frac{d s}{a^{2}(s)}, \quad K=\int_{0}^{1} \frac{d s}{a^{2}(s)}, \\
v(x)=\frac{y(\xi)}{a(\xi)}, \quad \rho(x)=\frac{\widehat{p}^{2} K^{2}}{L^{2}} a^{4}(\xi),
\end{gathered}
$$

and the Sturm-Liouville eigenvalue problem (3.3)-(3.4) (with $\widehat{q}(\xi)$ replaced by $q(\xi)$ ) is transformed back into the string eigenvalue problem

$$
\left\{\begin{array}{l}
\frac{d^{2} v(x)}{d x^{2}}+\lambda^{2} \rho(x) v(x)=0, \quad x \in(0, L), \\
v(0)=0=v(L) .
\end{array}\right.
$$

Therefore, the two strings $\{\widehat{\rho}(x)\},\{\rho(x)\}$ of equal length $L$, having fixed-fixed end conditions and pulled by unit tension, are quasi-isospectral. More precisely, given a number $n, n \geq 1$, we have $\lambda_{m}^{2}(\widehat{\rho}(x))=\lambda_{m}^{2}(\rho(x))$ for every $m \geq 1, m \neq n$, and the $n$th eigenvalue $\lambda_{n}^{2}(\rho(x))$ is connected with $\lambda_{n}^{2}(\widehat{\rho}(x))$ via (3.6). 


\subsection{Constructing beams with a given finite set of buckling loads.}

In this section we shall complete the proof of the main result of the paper.

Let us consider a P-P beam with $I_{0}=I_{0}(x)$ and buckling loads $\left\{\lambda_{m}^{2}\left(I_{0}\right)\right\}_{m=1}^{\infty}$ (e.g., eigenvalues of (2.3)-(2.5) with $I(x)$ replaced by $I_{0}(x)$ ). Starting from this P-P beam, we wish to construct a new P-P beam having prescribed values of the first $N, N \geq 1$, buckling loads $\left\{\widetilde{\lambda}_{m}^{2}\right\}_{m=1}^{N}$, with

$$
0<\widetilde{\lambda}_{1}^{2}<\widetilde{\lambda}_{2}^{2}<\ldots<\widetilde{\lambda}_{N}^{2} .
$$

Following the analysis of the previous sections, starting from the beam $I_{0}(x)$ we can construct a new beam $I_{1}(x)$ so that $\lambda_{m}^{2}\left(I_{1}\right)=\lambda_{m}^{2}\left(I_{0}\right)$ for $m \geq 2$, and $\lambda_{1}^{2}\left(I_{1}\right)$ coincides with the desired value $\widetilde{\lambda}_{1}^{2}$. More precisely, denoting by $a_{0}(\xi)$ the function $\widehat{a}(\xi)$ appearing in (3.2) (and corresponding to the initial beam $I_{0}(x)$ ), the function $a_{1}=a_{1}(\xi)$ associated to the new beam $I_{1}(x)$ is given by (3.21):

$$
a_{1}(\xi)=a_{0}(\xi)-t \frac{w_{1, t}(\xi)}{\mu_{1}\left(I_{0}\right) \omega_{1, t}(\xi)}\left[z_{1}\left(I_{0}\right), a_{0}\right](\xi)
$$

where the functions $w_{1, t}(\xi), \omega_{1, t}(\xi)$ are defined in (3.10)-(3.12), (3.13), respectively, with $\widehat{q}(\xi)$ replaced by $\widehat{q}_{0}(\xi)=\frac{1}{a_{0}(\xi)} \frac{d^{2} a_{0}(\xi)}{d \xi^{2}}$. Moreover, $\mu_{m}$ and $\lambda_{m}$ are linked as in (3.5), and $t$ satisfies (3.6). If $\widetilde{\mu}_{1}<\mu_{2}\left(I_{0}\right)$, then we can determine $t$, say $t=t_{1}$, such that $\mu_{1}\left(I_{1}\right)=\widetilde{\mu}_{1}$. The new beam $I_{1}(x)$ has buckling loads (or eigenvalues) $\left\{\widetilde{\lambda}_{1}^{2}, \lambda_{2}^{2}\left(I_{0}\right), \lambda_{3}^{2}\left(I_{0}\right), \ldots\right\}$, with $0<\widetilde{\lambda}_{1}^{2}<\lambda_{2}^{2}\left(I_{0}\right)<\lambda_{3}^{2}\left(I_{0}\right)<\ldots$, and can be used as starting point for the next step of the construction.

By repeating the above arguments, and provided that $\widetilde{\mu}_{2}<\mu_{3}\left(I_{0}\right)$, we can modify $I_{1}$ so as to keep $\lambda_{m}^{2}\left(I_{1}\right)$ fixed for $m \neq 2$ and move $\lambda_{2}^{2}\left(I_{1}\right)$ to the desired value $\widetilde{\lambda}_{2}^{2}$, by taking

$$
a_{2}(\xi)=a_{1}(\xi)-t_{2} \frac{w_{2, t_{2}}(\xi)}{\mu_{2}\left(I_{1}\right) \omega_{2, t_{2}}(\xi)}\left[z_{2}\left(I_{1}\right), a_{1}\right](\xi),
$$

where

$$
t_{2}=\widetilde{\mu}_{2}-\mu_{2}\left(I_{0}\right) .
$$

The buckling loads of the P-P beam $I_{2}(x)$ (associated to $a_{2}(\xi)$ ) are $\left\{\widetilde{\lambda}_{1}^{2}, \widetilde{\lambda}_{2}^{2}, \lambda_{3}^{2}\left(I_{0}\right), \lambda_{4}^{2}\left(I_{0}\right), \ldots\right\}$. By using repeatedly this procedure, after $N$ steps we construct a beam with coefficient $I_{N}(x)$ such that

$$
\lambda_{m}^{2}\left(I_{N}\right)=\widetilde{\lambda}_{m}^{2}, \quad \text { for } 1 \leq m \leq N,
$$

and the construction is completed. Clearly, the choice of the initial beam $I_{0}(x)$ is restricted by the conditions

$$
\widetilde{\lambda}_{1}^{2}<\lambda_{2}^{2}\left(I_{0}\right), \quad \widetilde{\lambda}_{2}^{2}<\lambda_{3}^{2}\left(I_{0}\right), \quad \ldots, \quad \widetilde{\lambda}_{N-1}^{2}<\lambda_{N}^{2}\left(I_{0}\right), \quad \widetilde{\lambda}_{N}^{2}<\lambda_{N+1}^{2}\left(I_{0}\right),
$$

which allow to determine uniquely the numbers $t_{1}, t_{2}, \ldots, t_{N}$ by expressions analogous to equation (3.29).

We notice that the above construction is not unique, since the flow from the initial beam $I_{0}$ to a beam with prescribed values of the first $N$ buckling loads 
depends on the particular order chosen to move every individual eigenvalue to the target value. As a consequence, the conditions (3.31) on the initial beam $I_{0}$ may change depending on the sequence of eigenvalue shifts.

Finally, we remark that previous arguments can be adapted to cover other sets of end conditions. In fact, by Proposition 2 of Ref. 12, the equivalence between the buckling problem for beams and the eigenvalue problem for strings stated in Proposition 2.1 can be extended to situations in which the beam, for example, has left end pinned and right end with a sliding constraint, e.g., $\frac{d v}{d x}(L)=0$ and $\frac{d}{d x}\left(I \frac{d^{2} v}{d x^{2}}\right)(L)=0$. The correspondence will link pinned and sliding end of the beam to fixed and free end of the string, respectively.

\section{Applications}

In this section we construct explicit examples of pinned-pinned beams which are quasi-isobuckling to the uniform beam, under the same end conditions, with $\widehat{I}(x)=$ $1, E=1$ and $L=1$. For a given number $n, n \geq 1$, the explicit expression (3.21) of $a(\xi)$ in $[0,1]$ takes the form

$$
a(\xi)=\beta \frac{\beta+(-1)^{n} \tan (n \pi \xi)\left[\tan \left(\beta n \pi\left(\xi-\frac{1}{2}\right)\right)\right]^{(-1)^{n}}}{1+\beta(-1)^{n} \tan (n \pi \xi)\left[\tan \left(\beta n \pi\left(\xi-\frac{1}{2}\right)\right)\right]^{(-1)^{n}}},
$$

with $\beta=\sqrt{\frac{t+(n \pi)^{2}}{(n \pi)^{2}}}$ and $t$ satisfying (3.6). Note that the function $a(\xi)$ is an even function with respect to the mid-point of the beam interval, and $a(0)=\beta^{2}=a(1)$.

The quasi-isobuckling beams shown in Figures 1-4 have been obtained for $n=1$ (Figures 1 and 2), $n=2$ (Figure 3) and $n=5$ (Figure 4), and for $\alpha=0.3,0.6,0.9$, where the shift parameter $\alpha \in[0,1)$ has been defined as $t=\alpha\left(\widehat{\lambda}_{n+1}^{2}-\widehat{\lambda}_{n}^{2}\right), t=$ $\alpha\left(\widehat{\lambda}_{n-1}^{2}-\widehat{\lambda}_{n}^{2}\right)\left(\widehat{\lambda}_{n}^{2}=(n \pi)^{2}, n \geq 0\right)$, for $t$ positive and $t$ negative, respectively.

It can be seen that when $\alpha$ approaches the limit value 1 (from the left), the coefficient $I(x)$ departs significantly from that of the uniform beam. In particular, in the case $n=1$ and for $t=\alpha\left(\widehat{\lambda}_{2}^{2}-\widehat{\lambda}_{1}^{2}\right)$, the bending stiffness is close to zero near the two ends of the beam and takes large values in the central part of the beam axis as $\alpha \rightarrow 1^{-}$(Figure 1b). Conversely, when $\alpha \rightarrow 1^{-}$and $t=-\alpha \widehat{\lambda}_{1}^{2}$, the coefficient $I(x)$ diverges as $x \rightarrow 0$ or $x \rightarrow 1$, whereas it vanishes as $x \rightarrow \frac{1}{2}$ (Figure 1 a and Figure 2). Figures 5 and 6 compare the first two buckling modes $v_{1}(x)$ (even) and $v_{2}(x)$ (odd) of the previous quasi-isobuckling beams with $n=1$. Each mode is normalized so that $\int_{0}^{1} \rho(x) v_{m}^{2}(x) d x=1, m=1,2$, and has been evaluated by means of the explicit expression (3.15). Mode shapes deviate significantly from those of the uniform beam when $\alpha$ is close to the limit value 1 .

The stiffness coefficient $I(x)$ shows a wavy behavior around the initial value $\widehat{I}=1$, with increasing oscillation as $n$ increases. For these situations, and for $t$ close to the limit values, the classical Euler-Bernoulli beam model is clearly not accurate and more sophisticate beam models would have to be considered. 
Figure 7 shows the plot of the global stiffness $F_{1}(t)=\int_{0}^{1} I(x, t) d x$ of our class of beams quasi-isobuckling to the uniform $P-P$ beam with $\widehat{I}=1, E=1, L=1$, for $n=1$. It turns out that $F_{1}(t)$ has a minimum exactly at $t=0$, which corresponds to the initial uniform beam.

Finally, the theoretical construction of quasi-isobuckling beams has been verified by finite element (FE) analysis. The discrete model herein adopted is based on a standard approximation of the transversal deflection $v=v(x)$ of the beam by means of third-order polynomial shape functions $\varphi_{k}=\varphi_{k}(x), k=1-4$, on a uniform mesh of $N_{e}$ equally spaced FEs. The stiffness coefficient is approximated by linear functions, that is $\widetilde{I}(x)=I\left(x_{e}\right)+\frac{I\left(x_{e+1}\right)-I\left(x_{e}\right)}{x_{e}+1-x_{e}} \cdot x$ within the eth finite element, $x \in\left[x_{e}, x_{e+1}\right]$. Therefore, the local 'inertia', $m_{e}^{i j}$, and stiffness, $k_{e}^{i j}$, matrix entries are given by

$$
m_{e}^{i j}=\int_{x_{e}}^{x_{e+1}} \varphi_{i}^{\prime}(x) \varphi_{j}^{\prime}(x) d x, \quad k_{e}^{i j}=\int_{x_{e}}^{x_{e+1}} \widetilde{I}(x) \varphi_{i}^{\prime \prime}(x) \varphi_{j}^{\prime \prime}(x) d x,
$$

$i, j=1-4$, and are evaluated in exact form. The value of the coefficient $I$ at a given node $x_{e}$ of the FE mesh is $I\left(x_{e}\right)=\rho^{-1}\left(x_{e}\right)$, for $\rho\left(x_{e}\right)=K^{2} a^{4}\left(\xi_{e}\right)$, where $\xi_{e}$ is the unique solution of the equation $x_{e}=\frac{1}{K} \int_{0}^{\xi_{e}} \frac{d s}{a^{2}(s)}, e=1, \ldots, N_{e}+1$ (with $x_{1}=0$, $x_{N_{e}+1}=1$ ). The discrete approximation of the continuous eigenvalue problem was solved by the Stodola-Vianello method.

Tables 1 and 2 compare the first 20 theoretical (exact) and numerical buckling loads for $n=1$ and a FE mesh with $N_{e}=100$. It can be seen that for $\alpha$ small enough the constructed beams are quasi-isospectral to the initial uniform beam within the accuracy of the FE approximation. Discrepancy increases as the shift parameter is close to the limit value (e.g., when $\alpha \rightarrow 1^{-}$) and as well as the mode order $m$ increases. As an example, Table 3 shows that a FE mesh with $N_{e}=200$ is enough to obtain a good approximation of the exact buckling loads for both Case (a) and Case (b). Finally, Table 4 suggests how to select the FE mesh dimension $N_{e}$ in order to maintain the average error on eigenvalues around 0.10 per cent. It can be seen that a value of $N_{e}$ equal to 10 or 50 times the corresponding value chosen to describe the uniform beam (100) is request to ensure errors of the same order for $\alpha \in(0.2,0.9)$.

\section{Conclusions}

In this paper we have considered the problem of constructing Euler-Bernoulli beams with prescribed values of the first $N$ buckling loads, under a specified set of boundary conditions. The key point of the procedure is the determination of quasiisobuckling beams, that is beams with different profile which have exactly the same buckling loads of an initial beam, with the exception of a given buckling load which is free to move in a prescribed interval. Quasi-isobuckling beams follow from the reduction of the buckling problem to an eigenvalue problem for the free vibration 
of a taut string, and on suitable application of a Darboux Lemma. The reconstruction procedure needs the specification of an initial beam whose buckling loads must satisfy certain interlacing conditions with the target buckling loads. Numerical simulations and comparison with finite element analysis support the theory.

\section{Funding}

This research received no specific grant from any funding agency in the public, commercial, or not-for-profit sectors.

\section{Acknowledgments}

The authors wish to thank Dr. Michele Dilena for providing the finite element code used in numerical simulations.

\section{Appendix A.}

In this appendix we recall the Darboux Lemma ${ }^{11}$.

Let $\mu$ be a real number, and suppose $g \equiv g(\xi)$ is a non-trivial solution of the Sturm-Liouville equation

$$
-g^{\prime \prime}+\widehat{q} g=\mu g
$$

with continuous potential $\widehat{q} \equiv \widehat{q}(\xi)$. If $f$ is a non-trivial solution of

$$
-f^{\prime \prime}+\widehat{q} f=\lambda f
$$

and $\lambda \neq \mu$, then

$$
y=\frac{1}{g}[g, f] \equiv \frac{1}{g}\left(g f^{\prime}-g^{\prime} f\right)
$$

is a non-trivial solution of the Sturm-Liouville equation

$$
-y^{\prime \prime}+\check{q} y=\lambda y,
$$

where

$$
\check{q}=\widehat{q}-2\left(\ln (g(\xi))^{\prime \prime} .\right.
$$

Moreover, the general solution of the equation

$$
-y^{\prime \prime}+\check{q} y=\mu y
$$

is

$$
y=\frac{1}{g}\left(b_{1}+b_{2} \int_{0}^{\xi} g^{2}(s) d s\right),
$$

where $b_{1}$ and $b_{2}$ are arbitrary constants. In particular, $y=\frac{1}{g}$ is a solution of (A.6).

It should be noted that if $g$ vanishes in [0,1], then equation (A.4) is understood to hold between the roots of $g$. These singular situations disappear by applying the Darboux Lemma twice. 


\section{References}

1. E.L. Nikolai, Lagrange's problem of optimal shape of a column, Izv. Leningr. Politekh. Inst. 255(8) (1907) (in Russian), reprinted in Works on Mechanics, G.I.T.T.L., Moskow (1955).

2. J.B. Keller, The shape of the strongest column, Archive for Rational Mechanics and Analysis 5 (1960) 275-285.

3. W. Prager, J.E. Taylor, Problems of optimal structural design, Journal of Applied Mechanics 34 (1968) 102-106.

4. N. Olhoff, S. Rasmussen, On single and bimodal optimum buckling loads of clamped columns, International Journal of Solids and Structures 13 (1977) 605-614.

5. D.C. Barnes, The shape of the strongest column is arbitrarily close to the shape of the weakest column, Quartely Applied Mathematics 41 (1988) 605-609.

6. S.J. Cox, M.L. Overton, On the optimal design of columns against buckling, SIAM Journal on Mathematical Analysis 40 (1992) 2532-2537.

7. I. Caliò, I. Elishakoff, Can a harmonic function constitute a closed-form buckling mode of an inhomogeneous column? AIAA Journal 40 (2002) 2532-2537.

8. I. Caliò, I. Elishakoff, Closed-form trigonometric solutions for inhomogeneous beam columns on elastic foundation, International Journal of Structural Stability and Dynamics 4(1) (2004) 139-146.

9. B. Yang, D.H. Park, Exact buckling analysis of constrained stepped columns, International Journal of Structural Stability and Dynamics 3 (2) (2003) 143-167.

10. A. Morassi, Exact construction of beams with a finite number of given natural frequencies, Journal of Vibration and Control 21 (2015) 591-600.

11. G. Darboux, Sur la répresentation sphérique des surfaces Comptes Rendus de l'Académie des Sciences 94 (1882) 1343-1345.

12. I. Caliò, G.M.L. Gladwell, A. Morassi, Families of beams with the same buckling spectrum Inverse Problems 27 (4) (2011), Paper 045006.

13. A.E.H. Love, A Treatise on the Mathematical Theory of Elasticity. (Dover Publications Edition, New York, 1944).

14. J. Pöschel, E. Trubowitz, Inverse Spectral Theory. (Academic Press, London, 1987). 


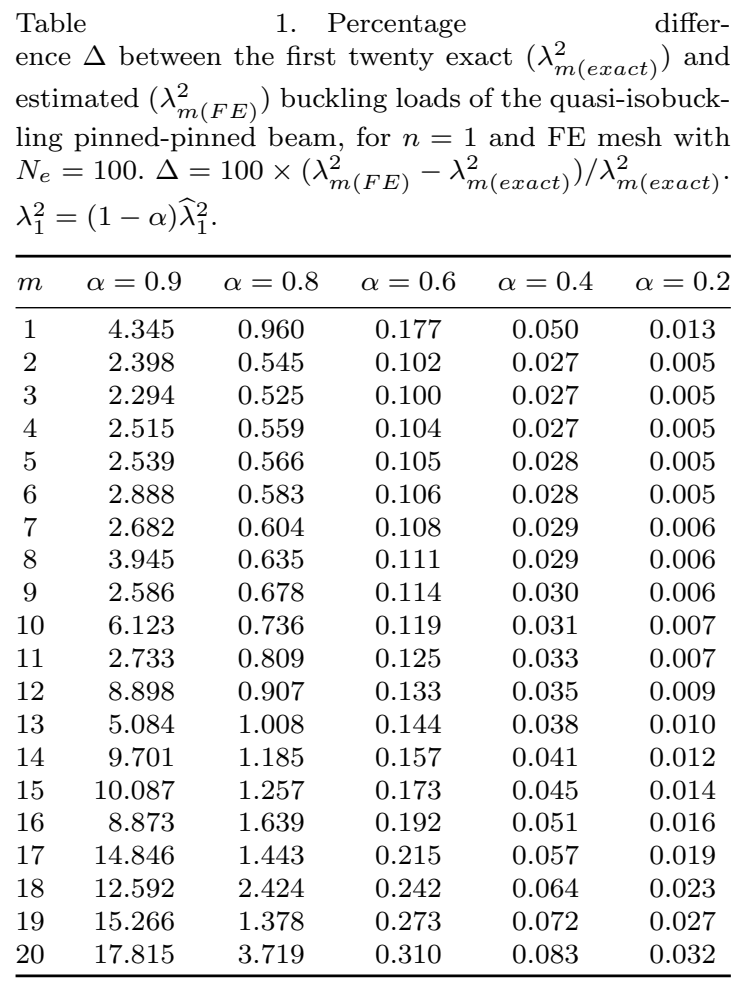


14 Morassi, Pressacco and Vrech

\begin{tabular}{|c|c|c|c|c|c|}
\hline \multicolumn{6}{|c|}{$\begin{array}{l}\text { Table } 2 . \text { Percentage } \\
\text { ence } \Delta \text { between the first twenty exact }\left(\lambda_{m(\text { exact })}^{2}\right) \text { and } \\
\text { estimated }\left(\lambda_{m(F E)}^{2}\right) \text { buckling loads of the quasi-isobuck- } \\
\text { ling pinned-pinned beam, for } n=1 \text { and FE mesh with } \\
N_{e}=100 . \Delta=100 \times\left(\lambda_{m(F E)}^{2}-\lambda_{m(\text { exact })}^{2}\right) / \lambda_{m(\text { exact })} \text {. } \\
\lambda_{1}^{2}=\widehat{\lambda}_{1}^{2}+\alpha\left(\widehat{\lambda}_{2}^{2}-\widehat{\lambda}_{1}^{2}\right) .\end{array}$} \\
\hline$m$ & $\alpha=0.2$ & $\alpha=0.4$ & $\alpha=0.6$ & $\alpha=0.8$ & $\alpha=0.9$ \\
\hline 1 & 0.013 & 0.142 & 1.091 & 15.377 & 70.101 \\
\hline 2 & & & & & 123.736 \\
\hline 3 & & & & & 66.431 \\
\hline 4 & & & & & 60.434 \\
\hline 5 & & 0.265 & & & 62.150 \\
\hline 6 & & 0269 & & 9 & 65.099 \\
\hline 7 & & & & & 68.096 \\
\hline 8 & & & & & 70.907 \\
\hline 9 & & & & & 73.468 \\
\hline 10 & & & & & 75.684 \\
\hline 11 & & 00 & & & 77.346 \\
\hline 12 & & & & & 78.146 \\
\hline 10 & & & & & 77.844 \\
\hline 14 & & & & 2 & 76.696 \\
\hline 15 & & & & 8 & 75.682 \\
\hline 16 & & & & & 75.899 \\
\hline 17 & & & & & 77.744 \\
\hline 18 & & & & & 80.934 \\
\hline 19 & & & & & 85.004 \\
\hline 20 & 0.101 & 0.556 & 1.796 & 25.231 & 89.578 \\
\hline
\end{tabular}


Table 3. Percentage difference $\Delta$ between the first twenty exact $\left(\lambda_{m(\text { exact })}^{2}\right)$ and estimated $\left(\lambda_{m(F E)}^{2}\right)$ buckling loads of the quasi-isobuckling pinned-pinned beam, for $n=1,2,5$ and $\mathrm{FE}$ mesh with $N_{e}=200 . \Delta=100 \times\left(\lambda_{m(F E)}^{2}-\lambda_{m(\text { exact })}^{2}\right) / \lambda_{m(\text { exact })}^{2}$. Case (a): $\lambda_{n}^{2}=\widehat{\lambda}_{n}^{2}-0.2\left(\widehat{\lambda}_{n}^{2}-\widehat{\lambda}_{n-1}^{2}\right)\left(\widehat{\lambda}_{0}^{2}=0\right)$. Case (b): $\lambda_{n}^{2}=\widehat{\lambda}_{n}^{2}+0.2\left(\widehat{\lambda}_{n+1}^{2}-\widehat{\lambda}_{n}^{2}\right)$.

\begin{tabular}{ccccccr}
\hline \multicolumn{3}{c}{ Case (a) } & \multicolumn{3}{c}{ Case (b) } \\
\hline $\mathrm{m}$ & $\mathrm{n}=1$ & $\mathrm{n}=2$ & $\mathrm{n}=5$ & $\mathrm{n}=1$ & $\mathrm{n}=2$ & $\mathrm{n}=5$ \\
\hline 1 & 0.003 & 0.004 & 0.005 & 0.003 & 0.008 & 0.006 \\
2 & 0.001 & 0.008 & 0.005 & 0.010 & -0.002 & 0.007 \\
3 & 0.001 & 0.003 & 0.005 & 0.010 & 0.006 & 0.007 \\
4 & 0.001 & 0.003 & 0.006 & 0.010 & 0.007 & 0.008 \\
5 & 0.001 & 0.003 & 0.019 & 0.010 & 0.007 & -0.012 \\
6 & 0.001 & 0.003 & 0.003 & 0.010 & 0.007 & 0.004 \\
7 & 0.001 & 0.003 & 0.003 & 0.011 & 0.007 & 0.005 \\
8 & 0.001 & 0.003 & 0.004 & 0.011 & 0.007 & 0.005 \\
9 & 0.001 & 0.003 & 0.004 & 0.011 & 0.007 & 0.005 \\
10 & 0.001 & 0.003 & 0.004 & 0.011 & 0.007 & 0.005 \\
11 & 0.001 & 0.003 & 0.004 & 0.011 & 0.007 & 0.006 \\
12 & 0.001 & 0.003 & 0.004 & 0.011 & 0.008 & 0.005 \\
13 & 0.002 & 0.003 & 0.004 & 0.011 & 0.008 & 0.006 \\
14 & 0.002 & 0.003 & 0.004 & 0.011 & 0.008 & 0.006 \\
15 & 0.002 & 0.004 & 0.004 & 0.012 & 0.008 & 0.006 \\
16 & 0.002 & 0.004 & 0.004 & 0.012 & 0.008 & 0.006 \\
17 & 0.002 & 0.004 & 0.005 & 0.012 & 0.008 & 0.006 \\
18 & 0.002 & 0.004 & 0.005 & 0.013 & 0.008 & 0.007 \\
19 & 0.003 & 0.004 & 0.005 & 0.014 & 0.008 & 0.007 \\
20 & 0.003 & 0.005 & 0.005 & 0.014 & 0.009 & 0.007 \\
\hline
\end{tabular}


16 Morassi, Pressacco and Vrech

Table 4. Percentage difference $\Delta$ between the first twenty exact $\left(\lambda_{m(\text { exact })}^{2}\right)$ and estimated $\left(\lambda_{m(F E)}^{2}\right)$ buckling loads of the quasi-isobuckling pinned-pinned beam, for $n=1$, varying the $\mathrm{FE}$ mesh size $N_{e}$. $\Delta=100 \times\left(\lambda_{m(F E)}^{2}-\lambda_{m(\text { exact })}^{2}\right) / \lambda_{m(\text { exact })}^{2}$. Case (a): $\lambda_{1}^{2}=\widehat{\lambda}_{1}^{2}-\alpha \widehat{\lambda}_{1}^{2}$. Case (b): $\lambda_{1}^{2}=\widehat{\lambda}_{1}^{2}+\alpha\left(\widehat{\lambda}_{2}^{2}-\widehat{\lambda}_{1}^{2}\right)$.

\begin{tabular}{ccccccccc}
\hline \multicolumn{7}{c}{ Case (a) } & & \multicolumn{3}{c}{ Case (b) } \\
\hline$\alpha$ & 0.9 & 0.8 & 0.6 & 0.4 & 0.4 & 0.6 & 0.8 & 0.9 \\
$N_{e}$ & 1000 & 500 & 200 & 100 & 200 & 500 & 2000 & 5000 \\
\hline 1 & 0.045 & 0.038 & 0.044 & 0.050 & 0.036 & 0.045 & 0.049 & 0.102 \\
2 & 0.024 & 0.022 & 0.025 & 0.027 & 0.070 & 0.072 & 0.064 & 0.118 \\
3 & 0.023 & 0.021 & 0.025 & 0.027 & 0.065 & 0.055 & 0.031 & 0.036 \\
4 & 0.024 & 0.022 & 0.026 & 0.027 & 0.065 & 0.057 & 0.034 & 0.038 \\
5 & 0.024 & 0.022 & 0.026 & 0.028 & 0.066 & 0.059 & 0.038 & 0.047 \\
6 & 0.025 & 0.022 & 0.026 & 0.028 & 0.067 & 0.061 & 0.040 & 0.050 \\
7 & 0.025 & 0.022 & 0.027 & 0.029 & 0.068 & 0.062 & 0.042 & 0.055 \\
8 & 0.025 & 0.023 & 0.027 & 0.029 & 0.068 & 0.063 & 0.043 & 0.058 \\
9 & 0.025 & 0.023 & 0.027 & 0.030 & 0.069 & 0.063 & 0.044 & 0.060 \\
10 & 0.025 & 0.023 & 0.027 & 0.031 & 0.069 & 0.063 & 0.044 & 0.061 \\
11 & 0.025 & 0.023 & 0.027 & 0.033 & 0.070 & 0.063 & 0.044 & 0.062 \\
12 & 0.025 & 0.023 & 0.028 & 0.035 & 0.071 & 0.064 & 0.044 & 0.062 \\
13 & 0.056 & 0.023 & 0.029 & 0.038 & 0.072 & 0.064 & 0.044 & 0.062 \\
14 & 0.026 & 0.024 & 0.030 & 0.041 & 0.073 & 0.064 & 0.044 & 0.061 \\
15 & 0.027 & 0.024 & 0.031 & 0.045 & 0.075 & 0.065 & 0.044 & 0.061 \\
16 & 0.027 & 0.025 & 0.032 & 0.051 & 0.076 & 0.065 & 0.044 & 0.061 \\
17 & 0.027 & 0.025 & 0.034 & 0.057 & 0.079 & 0.066 & 0.044 & 0.061 \\
18 & 0.028 & 0.026 & 0.036 & 0.064 & 0.081 & 0.066 & 0.044 & 0.061 \\
19 & 0.029 & 0.026 & 0.038 & 0.072 & 0.084 & 0.067 & 0.045 & 0.061 \\
20 & 0.030 & 0.027 & 0.040 & 0.083 & 0.088 & 0.068 & 0.045 & 0.061 \\
\hline & & & & & & & &
\end{tabular}




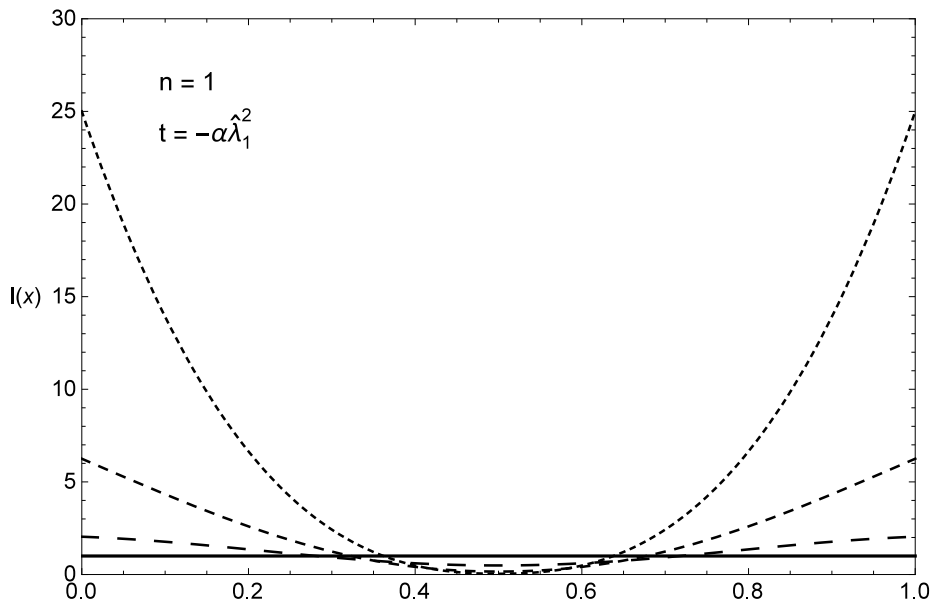

(a)

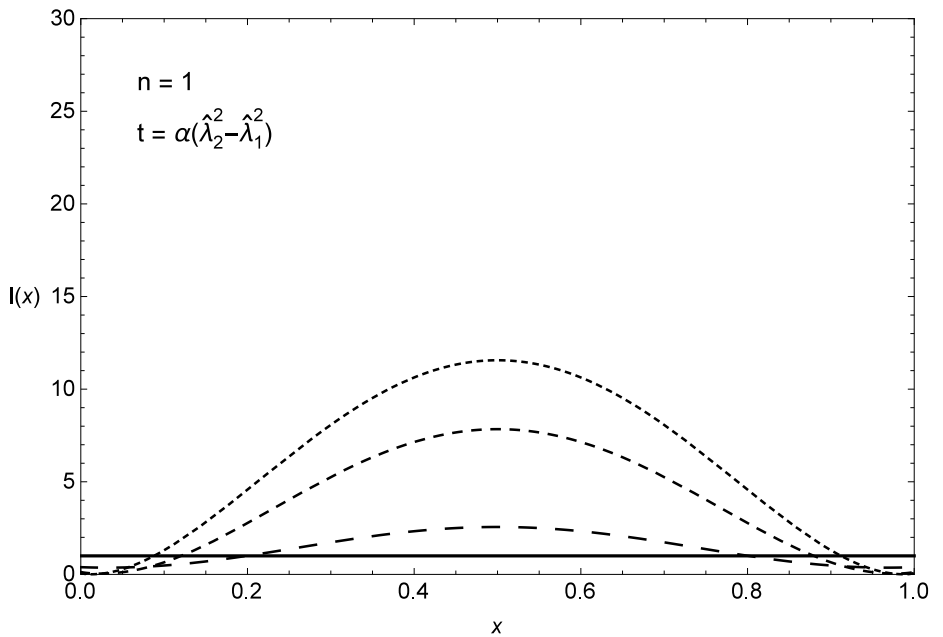

(b)

$$
\begin{aligned}
-\alpha & =0 \\
--\alpha & \alpha=0.6 \\
-\alpha & =0.3 \quad-\cdots \alpha=0.8
\end{aligned}
$$

Fig. 1. Examples of quasi-isobuckling pinned-pinned beams with $\widehat{I}(x)=1, E=1$ and $L=1$, for $n=1$ and $\alpha=0.3,0.6,0.8$. 


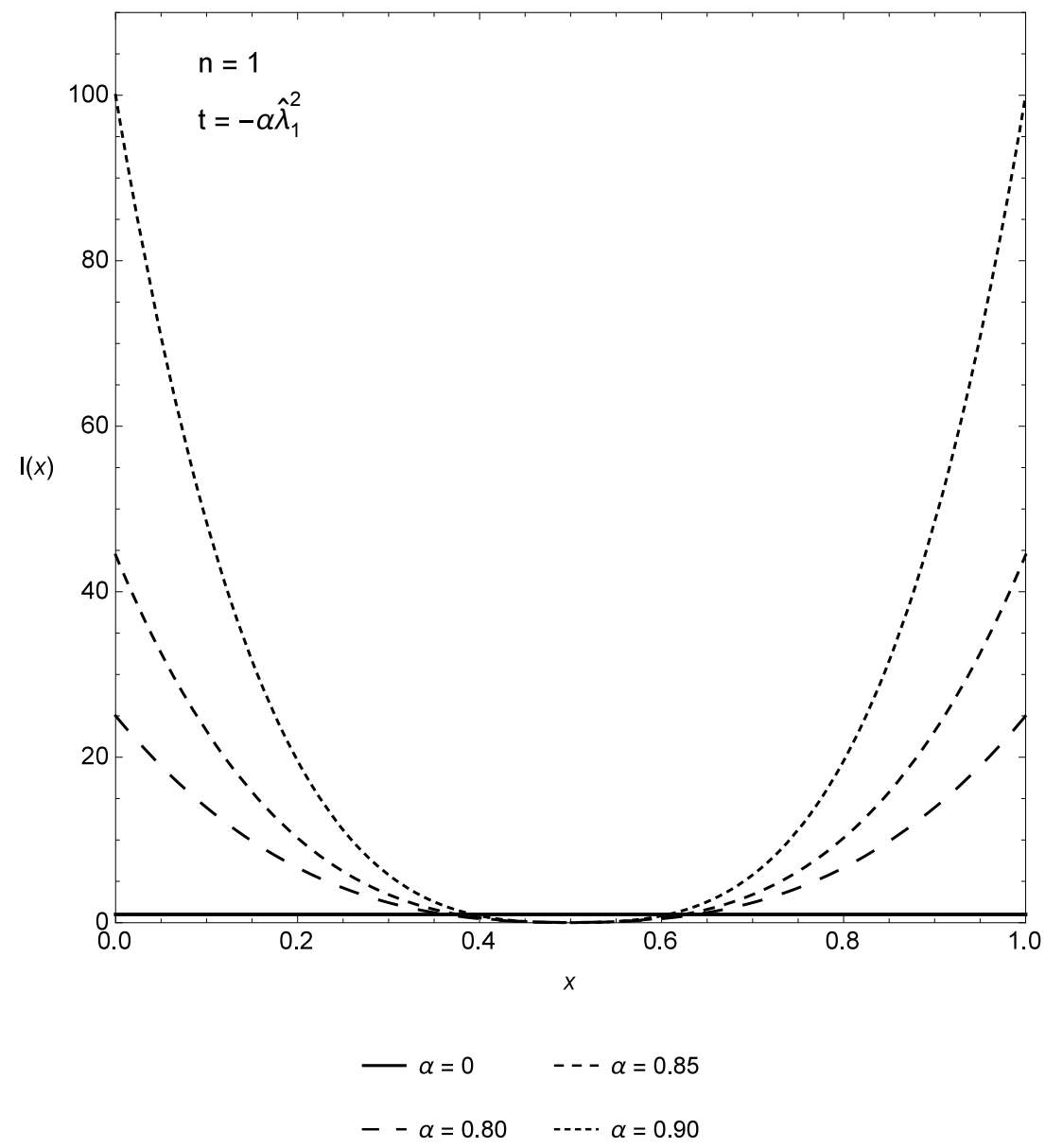

Fig. 2. Examples of quasi-isobuckling pinned-pinned beams with $\widehat{I}(x)=1, E=1$ and $L=1$, for $n=1$ and $\alpha=0.80,0.85,0.90$. 


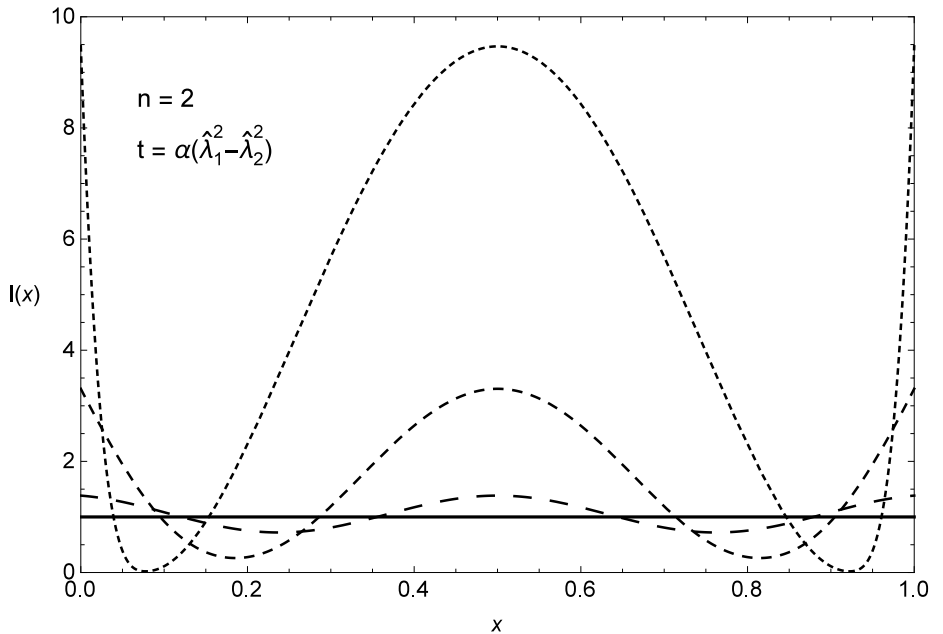

(a)

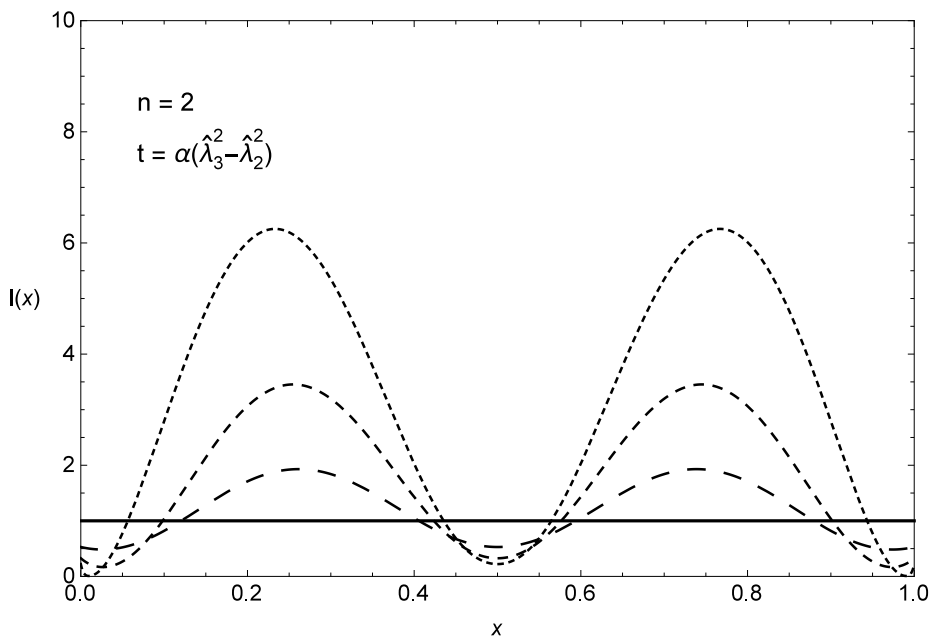

(b)

$$
\begin{aligned}
& -\alpha=0 \quad--\alpha=0.6 \\
& -\alpha=0.3 \quad \cdots \alpha=0.9
\end{aligned}
$$

Fig. 3. Examples of quasi-isobuckling pinned-pinned beams with $\widehat{I}(x)=1, E=1$ and $L=1$, for $n=2$ and $\alpha=0.3,0.6,0.9$. 


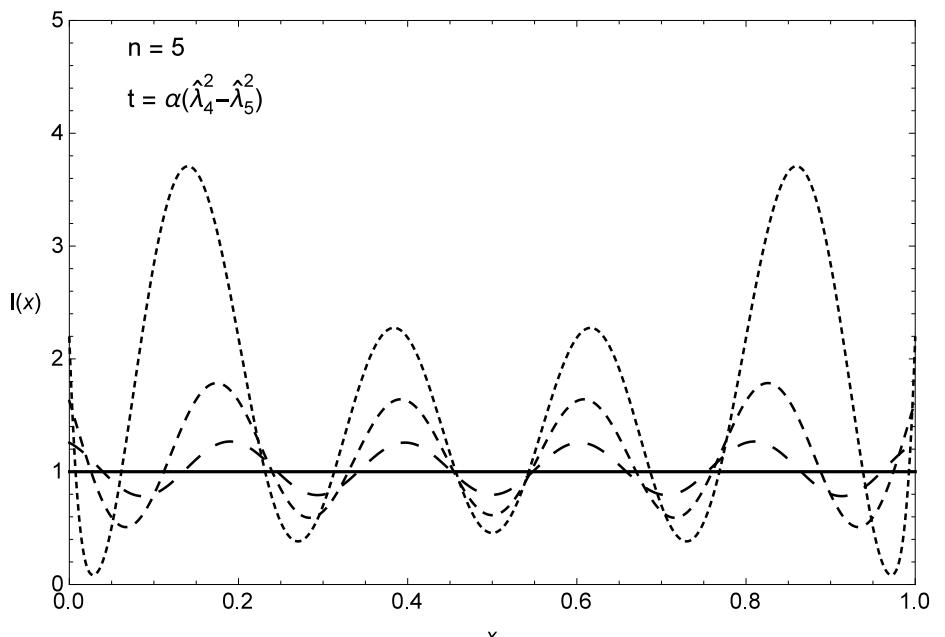

(a)

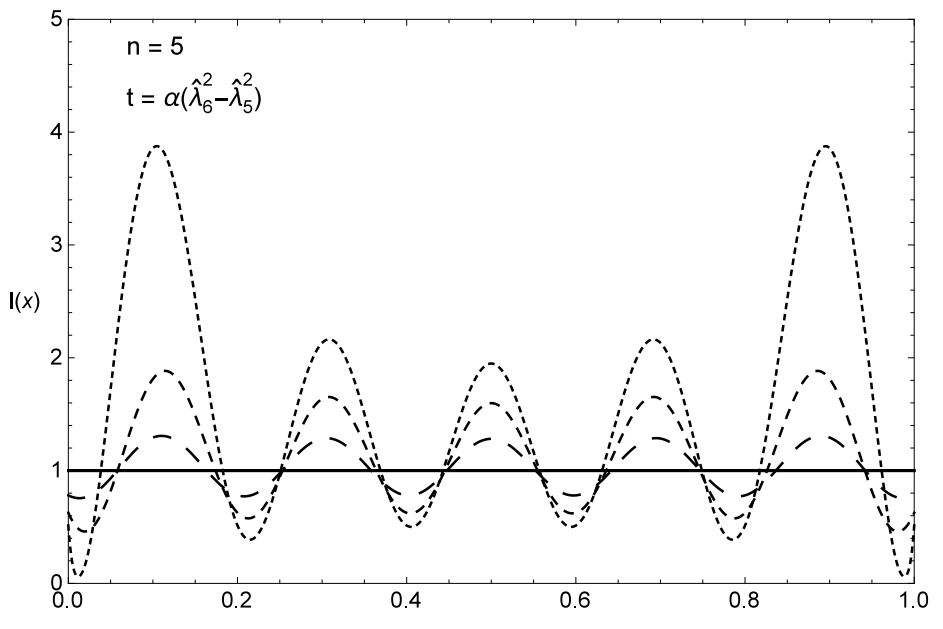

(b)

$$
\begin{aligned}
-\alpha & =0 \\
--\alpha & --\alpha=0.6 \\
-\cdots & \alpha=0.9
\end{aligned}
$$

Fig. 4. Examples of quasi-isobuckling pinned-pinned beams with $\widehat{I}(x)=1, E=1$ and $L=1$, for $n=5$ and $\alpha=0.3,0.6,0.9$. 


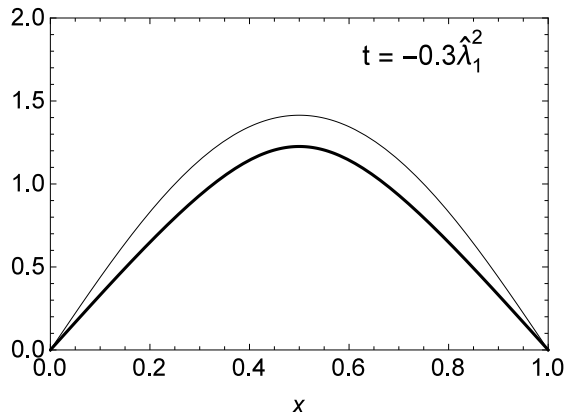

(a)

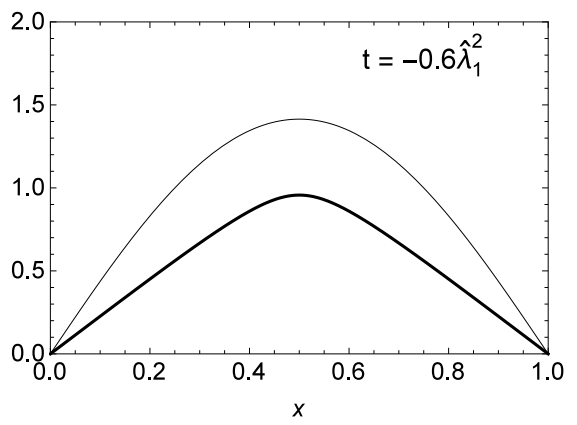

(c)

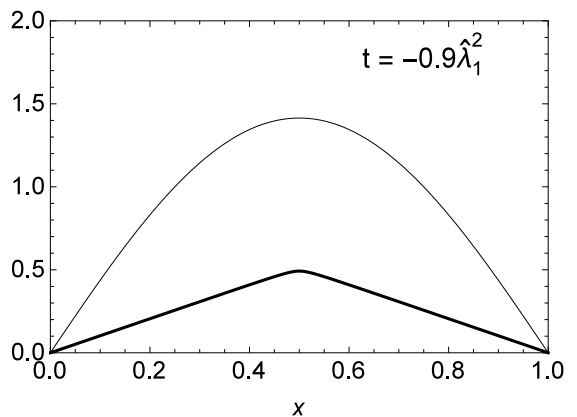

(e)

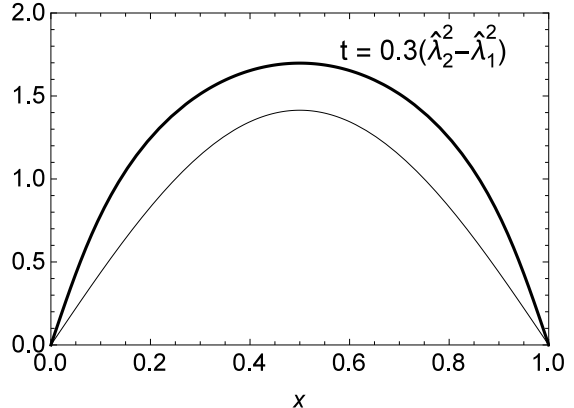

(b)

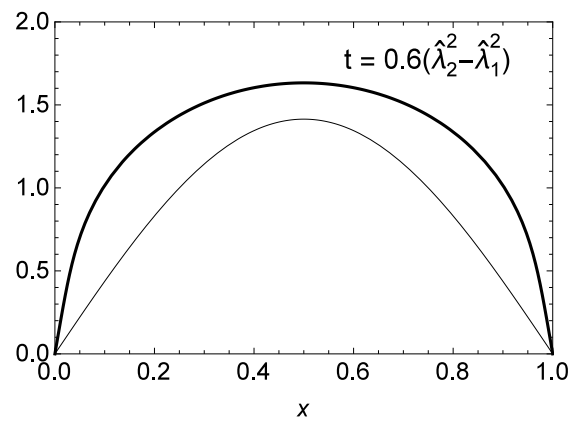

(d)

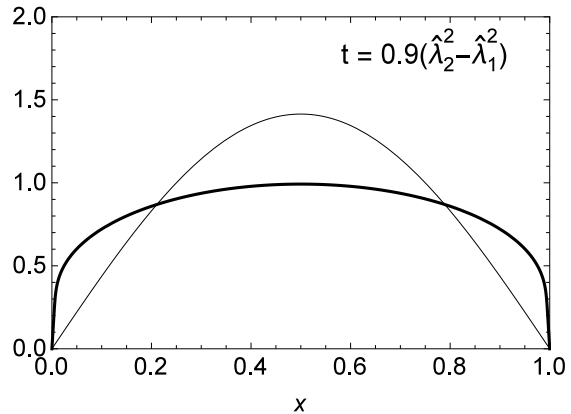

(f)

Fig. 5. Comparison of the first normalized buckling mode of the quasi-isobuckling P-P beam with negative (left column) and positive (right column) values of $t$, and $n=1$. $t=0$ corresponds to the uniform beam (thin line). 


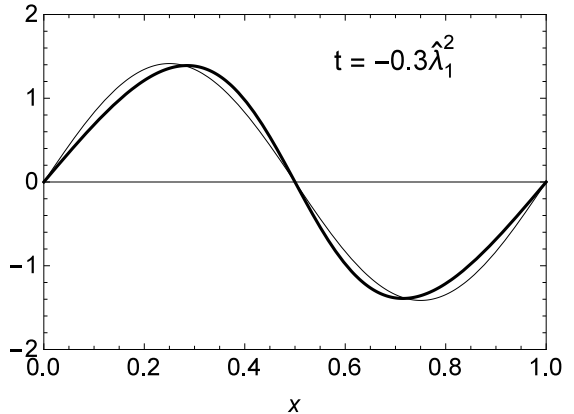

(a)

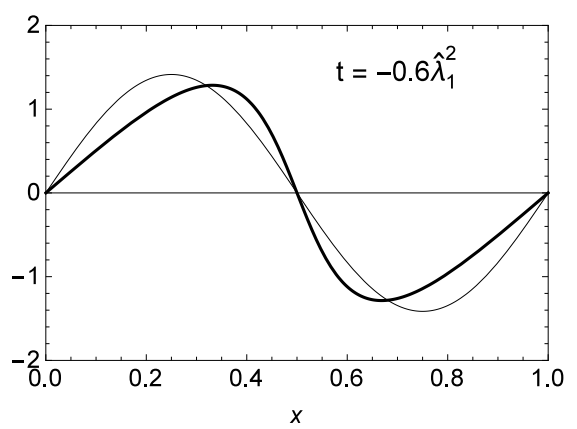

(c)

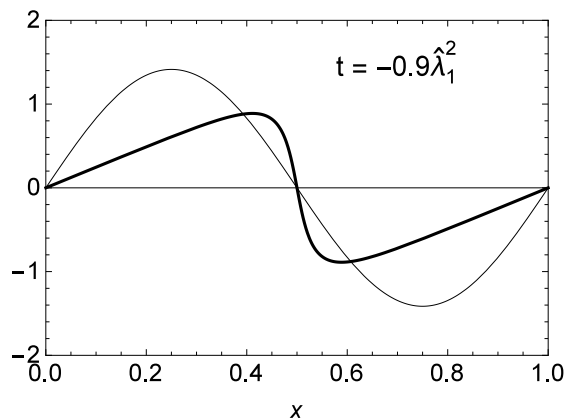

(e)

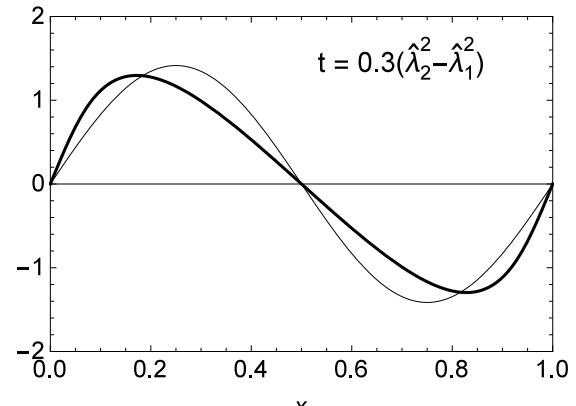

(b)

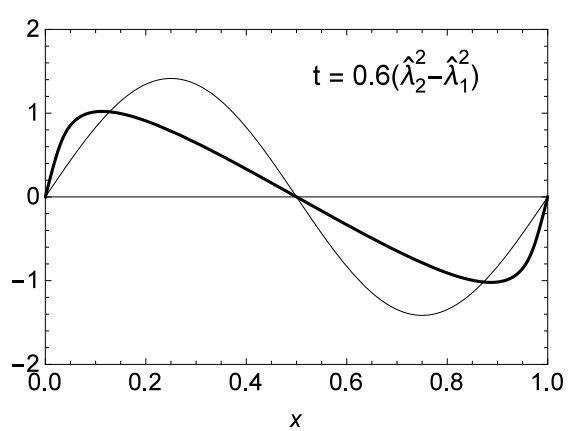

(d)

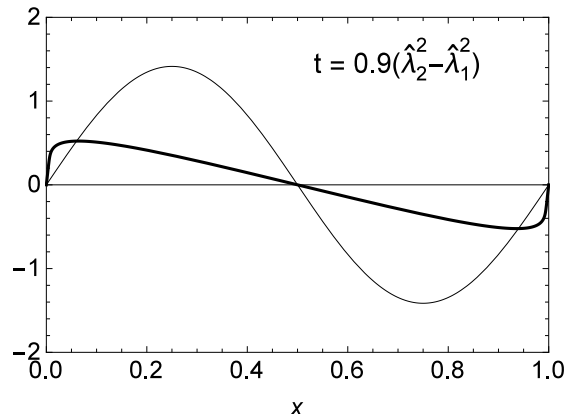

(f)

Fig. 6. Comparison of the second normalized buckling mode of the quasi-isobuckling P-P beam with negative (left column) and positive (right column) values of $t$, and $n=1 . t=0$ corresponds to the uniform beam (thin line). 
Explicit determination of pinned-pinned beams with a finite number of given buckling loads 23

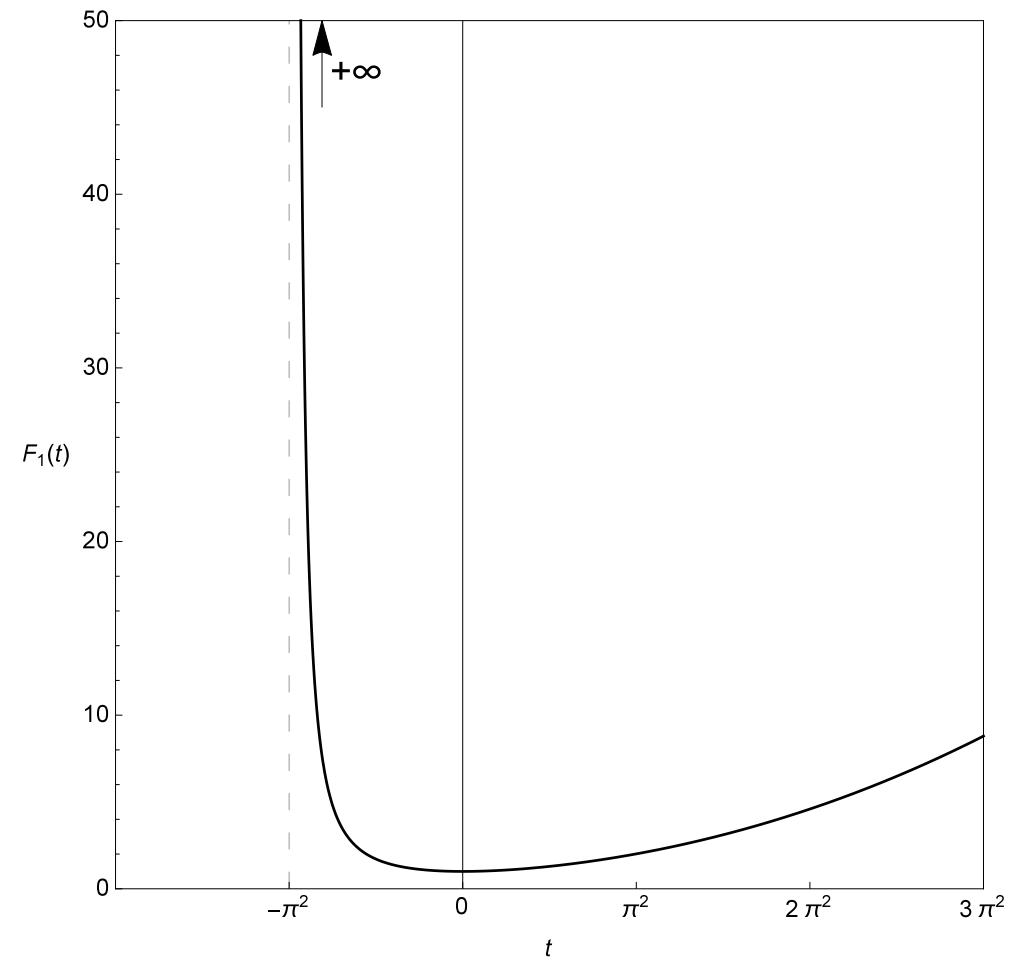

Fig. 7. Global stiffness $F_{1}(t)=\int_{0}^{1} I(x, t) d x$ for the quasi-isobuckling P-P beam, with $n=1$. 